\title{
ON THE NONCOMMUTATIVE GEOMETRY IN QUANTUM MECHANICS
}

\author{
Ilyas Haouam (iD \\ Laboratoire de Physique Mathématique et de Physique Subatomique (LPMPS), \\ Université Frères Mentouri, Constantine, Algeria
}

(Received 20 December 2019; in final form 12 March 2020; accepted 07 April 2020; published online 03 May 2020)

\begin{abstract}
In this paper, we presented and reviewed a formalism that plays a central role in most of the investigations concerning noncommutative geometry. We presented existing methods that successfully allow us to utilize and apply the noncommutativity of phase-space in both quantum mechanics and quantum field theory. In particular, we briefly explained the Weyl quantization, the Moyal-Weyl product, the Bopp-shift transformations, and the Seiberg-Witten maps.

Key words: noncommutative geometry, noncommutative quantum mechanics, Weyl quantization, Moyal-Weyl product, Bopp-shift transformation, Seiberg-Witten maps, Weyl-Wigner maps, uncertainty relations.
\end{abstract}

DOI: https://doi.org/10.30970/jps.24.2002

\section{INTRODUCTION}

The investigations of physics in noncommutative spaces recently have attracted a lot of attention not only because noncommutative space is necessary when considering the low-energy efficiency of D-brane with a background B-field, but also because in a tiny scale of strings or under very high energy conditions, effects of noncommutative space may appear. Besides one of the strongest motivations for noncommutative geometry is to obtain a coherent mathematical framework in which it would be possible to describe quantum gravitation.

Our purpose in this manuscript is to present the importance and the advantages of noncommutative geometry in both quantum mechanics and quantum field theory. The idea of the phase-space noncommutativity is strongly motivated in large part by the foundations of quantum mechanics in the context of canonical quantization. Furthermore, the origin of noncommutative geometry referring for the first time to the idea of noncommutative space-time was suggested by Heisenberg in 1930 and presented in 1947 by Snyder [1, 2] in view of the necessity to regularize the divergence of the quantum fields theory. This structure could appear as a natural generalization of the noncommutativity of phase-space in quantum mechanics. Also its origin was related to the search for topological spaces (when the commutative $\mathrm{C}^{*}$-algebras of functions are replaced by noncommutative functions algebras). Later A. Connes and others revived the concept of noncommutative geometry in 1985 [3-5]. They theorized the idea of a differential structure in a noncommutative framework by analyzing and defining the cyclic cohomology; in particular, they pointed out that the concept of differential calculus on manifolds had a noncommutative equivalent. Next, the noncommutative geometry got great support through many mathematical results, such as Theorem of Gelfand-Naïmark theorem on $\mathrm{C}^{*}$-algebras, cyclic cohomology of $C^{\infty}(M)$ algebra, $\mathrm{K}$ theory of $\mathrm{C}^{*}$ algebras, relations between Dirac operators and $\mathrm{Ri}$ emannian metrics, Serre-Swan theorem, and characterizations of commutative von Neumann algebras.
The noncommutative theory replaces the noncommutativity of operator associated with spacetime coordinates with deformation in the algebra of functions defined on space-time. On the other hand, a noncommutative version of a field theory is obtained by replacing the ordinary theory by a noncommutative theory, in other words, replacing the ordinary fields by noncommutative fields and the ordinary products by Moyal-Weyl products.

N. Seiberg and E. Witten published their famous article in 1999 [6], which was the most cited article according to Stanford Physics Information Retrieval System (SPIRES). This paper stimulated and encouraged great interest in noncommutative geometry, and became prevalent during the last years in the study of many physical problems. Then, it became clear that there is a close connection between noncommutative geometry and string theories. Studies of this geometric type and its implication contributed greatly to highlighting various fields of physics, particularly in matrix theory (also known as the matrix model BFSS (1997)), which is a tool for studying the properties of the M theory, it describes the behavior of a set of nine large matrices [7]. The noncommutative geometry is involved also in the description of quantum gravity theories, known as the noncommutative quantum field theories.

We present existing methods that successfully allow encoding and applying the noncommutativity of phasespace in quantum mechanics and quantum field theory, the Weyl quantization, the Moyal-Weyl product, the Bopp-shift transformations, and the Seiberg-Witten maps.

\section{CANONICAL FORMULATION OF NONCOMMUTATIVE GEOMETRY}

\section{A. The noncommutativity in phase-space}

The commutative algebra is an algebra where a product of two or more algebraic quantities does not depend on the order of the terms (i.e. $A B=B A$ ). 
For ordinary quantum mechanics, in the commutative phase-space, the coordinates $x_{j}$ and the momenta $p_{j}$ (the canonical variables) satisfy the usual canonical commutation relations

$$
\begin{aligned}
& {\left[x_{j}, x_{k}\right]=0} \\
& {\left[p_{j}, p_{k}\right]=0} \\
& {\left[x_{j}, p_{k}\right]=i \hbar \delta_{j k}}
\end{aligned}
$$

At string scales (very tiny scales), space no longer commutes, which allows us to admit the operators of coordinates and momenta in a $d$-dimensional noncommutative phase-space $x_{i}^{\text {nc }}$ and $p_{j}^{\text {nc }}$, respectively. The Heisenberg-like commutation relations are as follows

$$
\begin{aligned}
{\left[x_{j}^{\mathrm{nc}}, x_{k}^{\mathrm{nc}}\right] } & =i \Theta_{j k} \\
{\left[p_{j}^{\mathrm{nc}}, p_{k}^{\mathrm{nc}}\right] } & =i \eta_{j k} \quad(j, k=1, \ldots, d), \\
{\left[x_{j}^{\mathrm{nc}}, p_{k}^{\mathrm{nc}}\right] } & =i \hbar^{\mathrm{eff}} \delta_{j k}
\end{aligned}
$$

the effective Planck constant as shown in subsection VI A can be written as follows

$$
\hbar^{\mathrm{eff}}=\hbar(1+\xi),
$$

where

$$
\xi=\frac{\operatorname{Tr}[\Theta \eta]}{(2 \hbar)^{2}},
$$

and $\xi \ll 1$ is the condition of consistency in quantum mechanics. $\Theta_{j k}, \eta_{j k}$ are constant antisymmetric $d \times d$ matrices and $\delta_{j k}$ is the identity matrix. Theoretical predictions for noncommutative systems concerning noncommutative parameters as in the experience of Nesvizhevsky et al. $[8,9]$ showed that $\Theta \approx 10^{-30} \mathrm{~m}^{2}$ and $\eta \approx 1.76 \cdot 10^{-61} \mathrm{~kg}^{2} \mathrm{~m}^{2} \mathrm{~s}^{-2}$. Other bounds exist. For instance $\Theta \approx 4 \cdot 10^{-40} \mathrm{~m}^{2}$ when assuming $\hbar=c=1$ (the natural units) [10], and when taking into account that the experimental energy resolution is related to the uncertainty principle because of the finite lifetime of the neutron, this leads to obtaining $\eta \approx 10^{-67} \mathrm{~kg}^{2} \mathrm{~m}^{2} \mathrm{~s}^{-2}$.

These obtained results with that of the Nesvizhevsky et al. experiment allow us to estimate the consistency condition of the noncommutative model $|\xi| \leq 10^{-24}$. But if we consider the modifications introduced by the noncommutativity on the value of $\hbar$ which are at least about 24 orders of magnitude smaller than its value (with the precision of about $10^{-9}$ ), and considering the corrected bound of $\eta$, we have $|\xi| \leq 10^{-29}$ [11]. These values agree with the higher bounds on the basic scales of coordinate and momentum. These bounds will be suppressed if the used magnetic field in the experiment is weak $B \approx 5 \mathrm{mG}$.

In a 4-dimensional noncommutative space-time, we have the anti-commutation relations $[12,13]$

$$
\left[x_{\mathrm{nc}}^{\mu}, x_{\mathrm{nc}}^{\nu}\right]=i \Theta^{\mu \nu} \quad(\mu, \nu=0, \ldots, 3) .
$$

In the context of the field theory, it is more practical to introduce the noncommutative scale $\Lambda_{\mathrm{nc}}$ by

$$
\Theta^{\mu \nu}=\frac{c^{\mu \nu}}{\Lambda_{\mathrm{nc}}}
$$

where $c^{\mu \nu}$ is a dimensionless antisymmetric tensor, where the components are $\mathcal{O}(1)$. And $\Lambda_{\text {nc }}$ represents a characteristic energy scale for the noncommutative theory which is necessarily quite large.

For the noncommutative 3-dimensional systems, we have the following noncommutative algebra [14]

$$
\begin{aligned}
& {\left[x_{j}^{\mathrm{nc}}, x_{k}^{\mathrm{nc}}\right]=i \epsilon_{j k l} \Theta_{l}} \\
& {\left[p_{j}^{\mathrm{nc}}, p_{k}^{\mathrm{nc}}\right]=i \epsilon_{j k l} \eta_{l} \quad(j, k, l=1,2,3),} \\
& {\left[x_{j}^{\mathrm{nc}}, p_{k}^{\mathrm{nc}}\right]=i \hbar^{\mathrm{eff}} \delta_{j k}}
\end{aligned}
$$

$\Theta_{j k}, \eta_{j k}$ must be antisymmetric matrices of $(3 \times 3)$, and $\epsilon_{i j k}$ is the Levi-Civita symbol, and the summation convention is used. We have $\epsilon_{123}=\epsilon_{231}=\epsilon_{312}=$ $-\epsilon_{321}=-\epsilon_{132}=-\epsilon_{213}=1$, if $j=k$ or $k=l$, $\epsilon_{j k l}=0$. And $\Theta_{l}, \eta_{l}$ are the real-valued noncommutative parameters with the dimension of length ${ }^{2}$, momentum ${ }^{2}$ respectively. We neglect the relation of uncertainty between noncommutative canonical variables.

For the noncommutative 2-dimensional systems, we have the following noncommutative algebra

$$
\begin{aligned}
& {\left[x_{j}^{\mathrm{nc}}, x_{k}^{\mathrm{nc}}\right]=i \Theta \epsilon_{j k}} \\
& {\left[p_{j}^{\mathrm{nc}}, p_{k}^{\mathrm{nc}}\right]=i \eta \epsilon_{j k} \quad(j, k=1,2),} \\
& {\left[x_{j}^{\mathrm{nc}}, p_{k}^{\mathrm{nc}}\right]=i \hbar^{\mathrm{eff}} \delta_{j k}}
\end{aligned}
$$

we have $\epsilon_{12}=-\epsilon_{21}=1, \epsilon_{11}=\epsilon_{22}=0$, and $\Theta, \eta$ have real values and correspond to the components $\Theta_{12}$ and $\Theta_{21}$ of the noncommutative parameters in Eq. (2). Note that the relation (2) (according to the inequality of Cauchy-Schwarz) gives rise to the following phase-space uncertainty relation [13]

$$
(\Delta x)^{j}(\Delta x)^{k} \geq \frac{1}{2}\left|\Theta^{i j}\right|, \quad(\Delta p)^{j}(\Delta p)^{k} \geq \frac{1}{2}\left|\eta^{j k}\right| .
$$

\section{THE TIME-DEPENDENT NONCOMMUTATIVITY}

Several scientific works have focused on the timeindependent noncommutativity. The studies have considered experimentally the parameters of the noncommutativity of fixed values in the radiation frame of the cosmic microwave background, maybe considered approximately fixed to the celestial sphere, for instance, as proposed in the reference [15]. However, in this section, our obvious intention is to involve the time-dependency in noncommutative parameters. For instance, physical measurements must take into 
account the effect of the rotation of the Earth around its axis, which will introduce a time-dependency into the noncommutative parameters. Thus, we consider here a time-dependent noncommutative algebra, which satisfies the following commutation relations [16]

$$
\begin{aligned}
& {\left[x_{j}^{\mathrm{nc}}, x_{k}^{\mathrm{nc}}\right]=i \Theta_{j k}(t)} \\
& {\left[p_{j}^{\mathrm{nc}}, p_{k}^{\mathrm{nc}}\right]=i \eta_{j k}(t) \quad(j, k=1, \ldots, d),} \\
& {\left[x_{j}^{\mathrm{nc}}, p_{k}^{\mathrm{nc}}\right]=i \hbar^{\mathrm{eff}} \delta_{j k}}
\end{aligned}
$$

with

$$
\Theta_{j k}(t)=\Theta_{j k} e^{\gamma t} \quad \text { and } \quad \eta_{j k}(t)=\eta_{j k} e^{-\gamma t} .
$$

The position of the system cannot be localized in space, except for less infinite times. The space coordinates (the same for the momenta) become fuzzy and fluid [17]. The parameter $\Theta$ represents the fuzziness and the parameter $\gamma$ represents the fluidity of the space. The above relations are the known relations of the ordinary noncommutative geometry except that the parameters $\Theta(t), \eta(t)$ are considered exponential functi- ons. Of course, a multitude of other possibilities exists such as

$$
\Theta(t)=\Theta \cos (\gamma t), \quad \eta(t)=\eta \sin (\gamma t)
$$

\section{THE WEYL QUANTIZATION (WEYL OPERATOR)}

In 1927, Hermann K. H. Weyl introduced the quantization of Weyl, which is a technique used to describe quantum mechanics from the phase-space of classical mechanics. This elegant description allows us to associate a quantum observable with a real classical function that depends on the canonical variables on the phase space [18]. This technique provides a systematic way to describe non-commutative spaces in general and to study the theories of the fields defined on them. The condition of Schwartz $[19,20]$ equally implies that any function $f(x)$ defined on a Euclidean vector space $\mathcal{R}^{D}$ with $D$ dimensions can be described by its Fourier transform $\tilde{f}(k)$, by the relation

$$
\tilde{f}(k)=\int d^{D} x e^{-i k_{i} x^{i}} f(x), \quad \text { with } \quad \tilde{f}^{*}(k)=\tilde{f}(-k) \quad \text { if the function } f(x) \text { is real. }
$$

We define a noncommutative space-time by replacing the local coordinates $x_{i}$ of $\mathcal{R}^{D}$ by Hermitian operators $\hat{x}_{i}$, which verify the commutation relation (2). Then $\hat{x}_{i}$ generates a noncommutative algebra of operators.

Weyl's quantization consists in making a one-to-one correspondence between the algebra of functions $f(x)$ defined on $\mathcal{R}^{D}$ and the algebra of operators. Weyl's symbol is defined by [21]

$$
\hat{W}[f]=\int \frac{d^{D} k}{(2 \pi)^{D}} \tilde{f}(k) e^{i k_{i} \hat{x}^{i}}
$$

which is the operator field that corresponds to $f(x)$. Weyl's operator is Hermitian, if $f(x)$ is a real function

$$
\hat{W}^{\dagger}[f]=\hat{W}[f]
$$

\section{THE GROENEWOLD-MOYAL PRODUCT $(\star-$ PRODUCT)}

\section{A. Definition and formula}

The Moyal product (according to José E. Moyal) is also called the star product (noted $\star$ ) or the GroenewoldWeyl product (according to Hermann Weyl and Hilbrand
J. Groenewold). It is an associative deformation of the law of the product; it was first introduced during the development of the possible statistical signification of quantum mechanics and the relation between physical quantities and quantum mechanics operators.

The product of two Weyl operators of two functions provides the operator of Weyl associated with the star product of the two functions, i.e. using Eqs. (13-14) and according to the Baker-Campbell-Hausdorff $(\mathrm{BCH})$ formula $[22,23]$, we deduce that

$$
\hat{W}[f] \hat{W}[g]=\hat{W}[f \star g],
$$

where the Baker-Campbell-Hausdorff formula (the first few terms) is

$$
\begin{aligned}
e^{A} e^{B} & =\exp \left[A+B+\frac{1}{2}[A, B]\right. \\
& \left.+\frac{1}{12}([A,[A, B]]+[[A, B], B])+\ldots\right] .
\end{aligned}
$$

The noncommutative geometry is in turn described at the level of functions, actions and fields by the Gronewold-Moyal product. If the two functions vary in terms of $x$ and $p, f(x, p)$ and $g(x, p)$ are assumed to be infinitely differentiable, the Moyal product can be used as follows [24, 25] 


$$
\begin{aligned}
(f \star g)(x, p) & =\exp \left[\frac{i}{2} \Theta_{a b} \partial_{x_{a}} \partial_{x_{b}}+\frac{i}{2} \eta_{a b} \partial_{p_{a}} \partial_{p_{b}}\right] f\left(x_{a}, p_{a}\right) g\left(x_{b}, p_{b}\right)=f(x, p) g(x, p) \\
& +\sum_{n=1}\left(\frac{1}{n !}\right)\left(\frac{i}{2}\right)^{n} \Theta^{a_{1} b_{1}} \ldots \Theta^{a_{n} b_{n}} \partial_{a_{1}}^{x} \ldots \partial_{a_{k}}^{x} f(x, p) \partial_{b_{1}}^{x} \ldots \partial_{b_{k}}^{x} g(x, p) \\
& +\sum_{n=1}\left(\frac{1}{n !}\right)\left(\frac{i}{2}\right)^{n} \eta^{a_{1} b_{1}} \ldots \eta^{a_{n} b_{n}} \partial_{a_{1}}^{p} \ldots \partial_{a_{k}}^{p} f(x, p) \partial_{b_{1}}^{p} \ldots \partial_{b_{k}}^{p} g(x, p) .
\end{aligned}
$$

When the two functions vary in terms of $x$ only, then Eq. (18) is reduced to $[26,27]$

$$
\begin{aligned}
(f \star g)(x) & =\exp \left[\frac{i}{2} \Theta_{a b} \partial_{x_{a}} \partial_{x_{b}}\right] f\left(x_{a}\right) g\left(x_{b}\right)=f(x) g(x) \\
& +\sum_{n=1}\left(\frac{1}{n !}\right)\left(\frac{i}{2}\right)^{n} \Theta^{a_{1} b_{1}} \ldots \Theta^{a_{n} b_{n}} \partial_{a_{1}} \ldots \partial_{a_{k}} f(x) \partial_{b_{1}} \ldots \partial_{b_{k}} g(x) .
\end{aligned}
$$

The noncommutative field theories for slow-variation or low-energy fields $\left(\Theta E^{2} \lesssim 1\right)$ at the classical level are completely reduced to their commutative versions, because of the nature of the star product. But this is just the classical result and quantum corrections will reveal the influences of $\Theta$ even at low energies [28].

\section{Б. Some properties and rules}

Below we summarize some useful properties and identities [29] of the star product algebra. Let $f, g$ and $h$ be three arbitrary functions from $\mathcal{R}^{D}$

1. The star product is noncommutative

$$
f(x, p) \star g(x, p) \neq g(x, p) \star f(x, p), \quad \text { on the other hand } \quad f(x) \star g(x)=\left.g(x) \star f(x)\right|_{\Theta \rightarrow-\Theta},
$$

2. For $\Theta=\eta=0$, we find the commutative case

$$
\left.f(x, p) \star g(x, p)\right|_{\Theta=\eta=0}=f(x, p) g(x, p) .
$$

3. The complex conjugation

$$
(f(x) \star g(x))^{*}=g^{*}(x) \star f^{*}(x) .
$$

4. The star product between exponentials

$$
e^{i k x} \star e^{i q x}=e^{i(k+q) x} e^{-\frac{i}{2}(k \wedge q)}, \quad \text { with } \quad k \wedge q \equiv k^{\mu} q^{\nu} \theta_{\mu \nu} .
$$

5. Representation of momentum space

$$
f(x)=\int \tilde{f}(k) e^{i k x} d^{D} k, \quad g(x)=\int \tilde{g}(k) e^{i k x} d^{D} k, \quad \text { and } \quad(x)=\int \tilde{h}(k) e^{i k x} d^{D} k,
$$

where $\tilde{f}(k), \tilde{g}(k)$ and $\tilde{h}(k)$ are the Fourier transforms of the functions $f, g$ and $h$ respectively. Using Eq. (23) we find

$$
f(x) \star g(x)=\int \tilde{f}(k) \tilde{g}(q) e^{-i \frac{k \theta q}{2}} e^{i(k+q) x} d^{D} k d^{D} q .
$$


6. The associativity: Using the property (25) we find

$$
\begin{aligned}
& ((f \star g) \star h)(x)=\int \tilde{f}(k) \tilde{g}(q) \tilde{h}(p) e^{-i \frac{k \theta q}{2}} e^{-i \frac{(k+q) \theta p}{2}} e^{i(k+q+p) x} d^{D} k d^{D} q d^{D} p, \\
& (f \star(g \star h))(x)=\int \tilde{f}(k) \tilde{g}(q) \tilde{h}(p) e^{-i \frac{q \theta p}{2}} e^{-i \frac{(q+p) \theta k}{2}} e^{i(k+q+p) x} d^{D} k d^{D} q d^{D} p,
\end{aligned}
$$

the star product is associative

$$
(f(x) \star g(x)) \star h(x)=f(x) \star(g(x) \star h(x))=f(x) \star g(x) \star h(x) .
$$

7. The star product under the integral sign

$$
\int(f \star g)(x) d^{D} x=\int(g \star f)(x) d^{D} x=\int(f g)(x) d^{D} x,
$$

we learn that the kinetic part of the actions (which are quadratic in the fields) is the same as its commutative version. Thus, free-field propagators in commutative and noncommutative spaces are the same. This property follows for Schwartz functions on $\mathcal{R}^{D}$ via the integration by parts.

8. The integration over the space coordinates $x$ has the cyclic property and all the properties of the $\operatorname{Tr}$ in the matrix calculation

$$
\int(f \star g \star h)(x) d^{D} x=\int(h \star f \star g)(x) d^{D} x=\int(g \star h \star f)(x) d^{D} x .
$$

9. Leibniz's rule

$$
\partial_{\mu}(f \star g)=\left(\partial_{\mu} f\right) \star g+f \star\left(\partial_{\mu} g\right) .
$$

10. The Moyal commutator in terms of the first-order deformation quantization of $\Theta$

$$
[f, g]_{\star}=f \star g-g \star f=[f, g]+i \Theta^{i j} \partial_{i} f \partial g_{j}+0\left(\partial^{3} f, \partial^{3} g\right),
$$

for an Abelian group $([f, g]=0)$, the above equation becomes

$$
[f, g]_{\star}=i \Theta^{i j} \partial_{i} f \partial g_{j}+0\left(\partial^{3} f, \partial^{3} g\right) .
$$

An Abelian group (according to Niels Abel), also called a commutative group, is a group in which its internal composition law is commutative.

\section{THE SEIBERG-WITTEN (SW) MAPS}

The idea of the Seiberg-Witten maps has attracted a lot of attention and has been studied in depth in recent years, mainly on the basis that the theory of noncommutative gauges appears as a certain limit of the string theory. The relation to the string theory gives an interesting result: Noncommutative gauge theories can be mapped to commutative theories [6].

When you have a non-Abelian theory, such as the Yang-Mills theory(this type of theory was introduced in 1950 by C. N. Yang and R. Mills), the gauge structure will be encoded in commutators and anti-commutators. On the other hand, for an Abelian theory, all the commutators disappear. On the noncommutative side, the usual multiplication of functions is replaced by the star product (19), and we can write the star commutator to the first order by Eq. (32). The noncommutative Yang-Mills action is as follows [30]

$$
\begin{aligned}
S & =-\frac{1}{4} \operatorname{Tr} \int \hat{F}^{\mu \nu} \star \hat{F}_{\mu \nu} d^{4} k \\
& =-\frac{1}{4} \operatorname{Tr} \int \hat{F}^{\mu \nu} \hat{F}_{\mu \nu} d^{4} k,
\end{aligned}
$$

where the strength tensor of the non-commutative gauge field $\hat{A}_{\mu}$ is

$$
\hat{F}_{\mu \nu}=\partial_{\mu} \hat{A}_{\nu}-\hat{A}_{\mu} \partial_{\nu}-i\left[\hat{A}_{\mu}, \hat{A}_{\nu}\right]_{\star} .
$$

Equation (34) is invariant under the following noncommutative gauge transformations 


$$
\begin{aligned}
& \hat{\delta}_{\hat{\Lambda}} \hat{A}_{\mu}=\partial_{\mu} \hat{\Lambda}+i\left[\hat{\Lambda}, \hat{A}_{\mu}\right]_{\star} \equiv \hat{D}_{\mu} \hat{\Lambda}, \\
& \text { with } \\
& \hat{\delta}_{\hat{\Lambda}} \hat{F}_{\mu \nu}=i\left[\hat{\Lambda}, \hat{F}_{\mu \nu}\right]_{\star} .
\end{aligned}
$$

Here $\hat{\Lambda}$ is the gauge parameter in the noncommutative case. Take into consideration that for a commutative gauge theory, the potential four-vector of the gauge is $A_{\mu}$ (where the gauge parameter is $\Lambda$ ), with the gauge field strength tensor

$$
F_{\mu \nu}=\partial_{\mu} A_{\nu}-A_{\mu} \partial_{\nu}-i\left[A_{\mu}, A_{\nu}\right],
$$

we must differentiate the operators $\hat{A}, \hat{\Lambda}, \hat{\delta}, \ldots$ (with circumflex) and those without circumflex $A, \Lambda, \delta, \ldots$. Knowing that the transformations of the commutative gauge are

$$
\begin{aligned}
& \delta_{\Lambda} A_{\mu}=\partial_{\mu} \Lambda+i\left[\Lambda, A_{\mu}\right] \equiv D_{\mu} \Lambda, \\
& \text { with } \\
& \delta_{\Lambda} F_{\mu \nu}=i\left[\Lambda, F_{\mu \nu}\right] .
\end{aligned}
$$

The Seiberg-Witten maps make a connection (an equivalence relation) between a commutative and a noncommutative gauge theory. Such a relation between the noncommutative gauge theory and its ordinary (homologous) counterpart can also be demonstrated using only the algebraic structure of the aforementioned noncommutative space (canonically deformed) and the $\star$-product without reference to the string theory $[21,30,31]$. So it is possible to define an SW map that links as an equivalence relation of gauge by

$$
\hat{A}_{\mu}(A ; \Theta)+\hat{\delta}_{\hat{\Lambda}} \hat{A}_{\mu}(A ; \Theta)=\hat{A}_{\mu}\left(A+\delta_{\Lambda} A ; \Theta\right) .
$$

Note that the noncommutative gauge field $\hat{A}_{\mu}$ and the noncommutative gauge parameter $\hat{\Lambda}$ have the following functional dependency (i.e. one can consider them as functional ordinary fields $A_{\mu}, F_{\mu \nu}$ )

$$
\begin{aligned}
& \hat{A}_{\mu}=\hat{A}_{\mu}(A ; \Theta), \quad \hat{F}_{\mu \nu}=\hat{F}_{\mu \nu}(A ; \Theta), \\
& \text { and } \\
& \hat{\Lambda}=\hat{\Lambda}(\hat{\Lambda}, A ; \Theta) .
\end{aligned}
$$

The transformation comes down to the following diagram (according to [32])

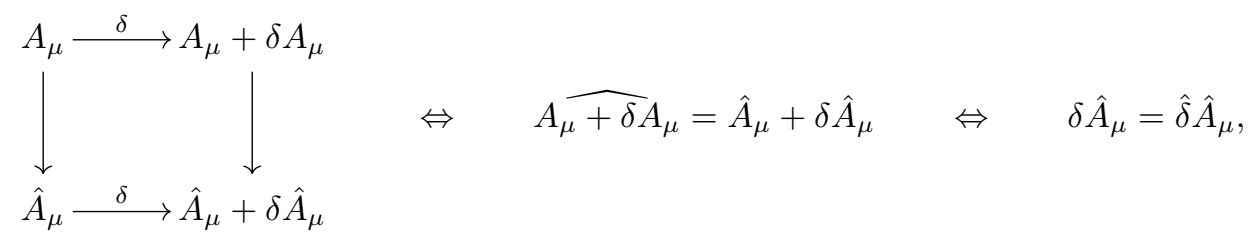

where it is equivalent to transforming function $A_{\mu}$ by $\delta$ and to transforming the noncommutative connection $\hat{A}_{\mu}$ by $\hat{\delta}$. This is what we will call the Seiberg-Witten equation

$$
\delta_{\Lambda} \hat{A}_{\mu}-\partial_{\mu} \hat{\Lambda}=i\left[\hat{\Lambda}, \hat{A}_{\mu}\right]_{\star} .
$$

To solve Eq. (39), we use the condition of consistency(coherence) of noncommutative gauge [30]

$$
i \hat{\delta}_{\alpha} \hat{\Lambda}_{\beta}-i \hat{\delta}_{\beta} \hat{\Lambda}_{\alpha}-\left[\hat{\Lambda}_{\alpha}, \hat{A}_{\beta}\right]_{\star}=i \hat{\Lambda}_{-i[\alpha, \beta]},
$$

and its ordinary counterpart given by

$$
\delta_{\alpha} \delta_{\beta}-\delta_{\beta} \delta_{\alpha}=\delta_{-i[\alpha, \beta]}=\delta_{\alpha \times \beta},
$$

where $\alpha, \beta$ are Hermitian matrices. Take into account that for the components of order $n$ of $\hat{\Lambda}_{\alpha}$ and $\hat{\Lambda}_{\beta}$, it is then possible to write a coherence equation of noncommutative gauge

$$
i \hat{\delta}_{\alpha} \hat{\Lambda}_{\beta}^{n}-i \hat{\delta}_{\beta} \hat{\Lambda}_{\alpha}^{n}-\sum_{p+q+r=n}\left[\hat{\Lambda}_{\alpha}^{p}, \hat{A}_{\beta}^{q}\right]_{\star^{r}}=i \hat{\Lambda}_{-i[\alpha, \beta]}^{n},
$$

and $\star^{r}$ denotes

$$
\begin{aligned}
& \left(f \star^{r} g\right)(x)=\frac{1}{r !}\left(\frac{i}{2}\right)^{r} \\
& \times \Theta^{a_{1} b_{1}} \ldots \Theta^{a_{r} b_{r}} \partial_{a_{1}} \ldots \partial_{a_{r}} f(x) \partial_{b_{1}} \ldots \partial_{b_{r}} g(x) .
\end{aligned}
$$

Consequently,

$$
\begin{aligned}
& \alpha \times \beta=-i[\alpha, \beta]=\alpha_{a} \beta_{b} f_{c}^{a b} T^{c}, \\
& \text { with } \\
& f_{c}^{a b} T^{c}=-i\left[T^{a}, T^{b}\right],
\end{aligned}
$$

where $T^{a}$ are representations of a group $S U(N)$, consequently, a non-Abelian gauge theory is based on 
this Lie algebra. More precisely, we write $\alpha, \beta$ from $A_{x}$, which form a representation of the T-algebra (the generators $T$ of the Lie algebra). Knowing that $A_{x} \equiv$ $\mathbb{C}\left[\left[\hat{x}^{1}, \ldots, \hat{x}^{N}\right]\right] / \mathcal{R}$, in which the non-commutative space can be defined as the associative algebra over $\mathbb{C}$, which consists of the algebra freely generated by the coordinates and then divided by the ideal $\mathcal{R}$. For the components of order $n$ of $\Lambda_{\alpha}$ and $\Lambda_{\nu}$, it is also possible to write a gauge consistency equation

$$
\delta_{\alpha} \Lambda_{\nu}^{n}=\partial_{\nu} \Lambda_{\alpha}^{n}-\sum_{p+q+r=n}\left[\Lambda_{\nu}^{p}, \Lambda_{\alpha}^{q}\right]_{\star^{r}}
$$

To find SW maps of the noncommutative gauge parameter $\hat{\Lambda}$ and the field of the noncommutative gauge $\hat{A}_{\mu}$, we can solve the condition of consistency (42) and the equivalence gauge (39). For this purpose, noncommutative variables can be extended as a series of formal powers in $\Theta$

$$
\begin{aligned}
\hat{A}_{\mu} & =A_{\mu}+A_{\mu}^{(1)}+A_{\mu}^{(2)} \ldots, \\
\hat{F}_{\mu \nu} & =F_{\mu \nu}+F_{\mu \nu}^{(1)}+F_{\mu \nu}^{(2)}+\ldots, \\
\hat{\Lambda} & =\Lambda+\Lambda^{(1)}+\Lambda^{(2)}+\ldots,
\end{aligned}
$$

the terms of zero-order are ordinary counterparts. Moreover, allowing the theory to be a valued enveloping algebra, we can construct the noncommutative gauge theory for an arbitrary gauge group such as $S U(N)$. Furthermore, we will not specify the gauge group and the results will be valid for a group of an arbitrary nonAbelian gauge.

Thus, we have the first-order solution given in the original document [3]

$$
\begin{aligned}
& \Lambda_{\alpha}^{(1)}=-\frac{1}{4} \Theta^{k \lambda}\left\{A_{k}, \partial_{\lambda} \alpha\right\}, \\
& \Lambda_{\gamma}^{(1)}=-\frac{1}{4} \Theta^{k \lambda}\left\{A_{k}, \partial_{\lambda} A_{\gamma}+F_{\lambda k}\right\},
\end{aligned}
$$

where $\partial_{\lambda} \alpha=\delta_{\alpha} A_{\lambda}-i\left[\alpha, A_{\lambda}\right]$. We can also find the strength tensor of the first-order field (35)

$$
\begin{aligned}
F_{\gamma \rho}^{(1)}= & -\frac{1}{4} \Theta^{k \lambda}\left(\left\{A_{k}, \partial_{\lambda} F_{\gamma \rho}+D_{\lambda} F_{\gamma \rho}\right\}\right. \\
& \left.-2\left\{F_{\gamma k}, F_{\rho \lambda}\right\}\right),
\end{aligned}
$$

where the products on the right hand side in the above equations, such as $\left\{A_{k}, \partial_{\lambda} \alpha\right\}=A_{k} . \partial_{\lambda} \alpha+\partial_{\lambda} \alpha . A_{k}$ are ordinary matrix products.

It should be emphasized here once again that these solutions are not unique because one can add homogeneous solutions to (49) with arbitrary coefficients. However, the structure of these solutions will be useful to obtain recursive solutions to all orders.

Indeed, the explicit solutions for SW maps of the non-Abelian gauge theory have been found by various authors until the second-order of $\Theta[30,33,34]$. Because of the liberty in the solutions (49), these maps are different from each other by a homogeneous solution with different coefficients. Finally, explicit SeibergWitten maps of noncommutative fields obviously are necessary to understand physical predictions and to verify the behavior of the noncommutative theory itself, such as the renormalizability.

\section{THE BOPP-SHIFT LINEAR TRANSLATION}

\section{A. The Bopp-shift translation}

In the noncommutative phase-space, coordinates $x_{i}^{\mathrm{nc}}$ and momenta $p_{i}^{\text {nc }}$ satisfy the commutation relations (2), and using equations (1-2), we can represent variables $x_{i}^{\text {nc }}$ and $p_{i}^{\text {nc }}$ in terms of $x_{i}$ and $p_{i}$ by $[35,36]$

$$
\begin{aligned}
& x_{i}^{\mathrm{nc}}=a_{i j} x_{j}+b_{i j} p_{j} \text { and } p_{i}^{\mathrm{nc}}=c_{i j} x_{j}+d_{i j} p_{j} \\
& \text { with } \\
& i, j=1, \ldots, n .
\end{aligned}
$$

The system of equations (51) can also be rewritten as follows

$$
\left(\begin{array}{l}
x^{\mathrm{nc}} \\
p^{\mathrm{nc}}
\end{array}\right)=\left(\begin{array}{cc}
A & B \\
C & D
\end{array}\right)\left(\begin{array}{l}
x \\
p
\end{array}\right),
$$

where $A=\left(a_{i j}\right), B=\left(b_{i j}\right)$, and $C=\left(c_{i j}\right), D=\left(d_{i j}\right)$ aresquare matrices of $n \times n$ dimension.

We calculate the exact form of the above matrices. For this, we use equations (1-2 and 51), we obtain the following system of equation

$$
\begin{aligned}
& A B^{T}-B A^{T}=\frac{\Theta}{\hbar} \\
& C D^{T}-D C^{T}=\frac{\eta}{\hbar} \\
& A D^{T}-B C^{T}=\frac{\hbar^{\text {eff }}}{\hbar} I_{n \times n} .
\end{aligned}
$$

Matrices $A$ and $D$ can be chosen proportionally to the identity operator, which we respectively designate by $\alpha$ and $\beta$, which means $a_{i j}=\alpha \delta_{i j}$ and $d_{i j}=\beta \delta_{i j}$ where $\alpha$ and $\beta$ are two scaling constants. Thus, the system becomes

$$
\begin{aligned}
& \alpha\left(B^{T}-B\right)=\Theta \\
& \beta\left(C-C^{T}\right)=\eta \\
& B C^{T}=(\alpha \beta-1) I_{n \times n}
\end{aligned}
$$

according to the first two equations in Eq. (54), we can see that, if $B, C$ are antisymmetric, they will have explicit solutions. The third equation in Eq. (54) is satisfied only when $B, C$ are symmetric or antisymmetric. The symmetrical case of $B, C$ leads to $\Theta=\eta=0$ (which 
means that our space is commutative). So we choose $B$, $C$ antisymmetric, and we find

$$
B=-\frac{1}{2 \alpha \hbar} \Theta, \quad C=\frac{1}{2 \beta \hbar} \eta
$$

By substituting the expressions of $A, B, C$ and $D$ in the third equation of Eq. (53), we get the generalized expression of the effective Planck constant

$$
\hbar^{\mathrm{eff}}=\alpha \beta \hbar+\frac{\operatorname{Tr}[\Theta \eta]}{4 \hbar \alpha \beta}
$$

We insert Eq. (55) in Eq. (52) and obtain

$$
\left(\begin{array}{l}
x^{\mathrm{nc}} \\
p^{\mathrm{nc}}
\end{array}\right)=\left(\begin{array}{cc}
\alpha I_{n \times n} & -\frac{1}{2 \alpha \hbar} \Theta \\
\frac{1}{2 \beta \hbar} \eta & \beta I_{n \times n}
\end{array}\right)\left(\begin{array}{l}
x \\
p
\end{array}\right),
$$

so Eq. (51) becomes [37]

$$
x_{i}^{\mathrm{nc}}=\alpha x_{i}-\frac{1}{2 \alpha \hbar} \Theta_{i j} p_{j}, \quad p_{i}^{\mathrm{nc}}=\beta p_{i}+\frac{1}{2 \beta \hbar} \eta_{i j} x_{j}, \quad \text { with } \quad i, j=1,2 \ldots, n .
$$

The above equations (58) are the generalized Bopp-shift transformation, with $\alpha=1-\frac{\Theta \eta}{8 \hbar^{2}}$ and $\beta=\frac{1}{\alpha}$. Generally in the calculations, we take $\alpha=\beta=1$, so Eqs. $(58,56)$ become

$$
x_{i}^{\mathrm{nc}}=x_{i}-\frac{1}{2 \hbar} \Theta_{i j} p_{j}, \quad p_{i}^{\mathrm{nc}}=p_{i}+\frac{1}{2 \hbar} \eta_{i j} x_{j} \text { and } \hbar^{\mathrm{eff}}=\hbar+\frac{\Theta \eta}{4 \hbar}, \text { with } i, j=1,2, \ldots, n .
$$

When we consider a 2-dimensional non-commutative phase-space $(i, j=1,2)$, we have

$$
\begin{aligned}
& \text { For } \quad i=1, \quad x_{1}^{\mathrm{nc}}=x^{\mathrm{nc}}=x-\frac{1}{2 \hbar} \Theta p_{y}, \quad p_{1}^{\mathrm{nc}}=p_{x}^{\mathrm{nc}}=p_{x}+\frac{1}{2 \hbar} \eta y . \\
& \text { For } i=2, \quad x_{2}^{\mathrm{nc}}=y^{\mathrm{nc}}=y+\frac{1}{2 \hbar} \Theta p_{x}, \quad p_{2}^{\mathrm{nc}}=p_{y}^{\mathrm{nc}}=p_{y}-\frac{1}{2 \hbar} \eta x,
\end{aligned}
$$

so finally, we find the following Darboux transformation

$$
\begin{aligned}
& x^{\mathrm{nc}}=x-\frac{1}{2 \hbar} \Theta p_{y}, \quad p_{x}^{\mathrm{nc}}=p_{x}+\frac{1}{2 \hbar} \eta y \\
& y^{\mathrm{nc}}=y+\frac{1}{2 \hbar} \Theta p_{x}, \quad p_{y}^{\mathrm{nc}}=p_{y}-\frac{1}{2 \hbar} \eta x .
\end{aligned}
$$

We can say that the operators of noncommutativity in phase-space are linked to the commutative operators by the Heisenberg-Weyl algebra in terms of the Bopp-shift linear translation introduced from Eq. (62).

\section{Б. Time-dependent Bopp-shift translation}

The new deformed geometry of time-dependent noncommutativity in phase-space is linked to commutative geometry by the Heisenberg-Weyl algebra via the time-dependent linear Bopp-shift linear transformation introduced from the equation

$$
x_{i}^{\mathrm{nc}}(t)=x_{i}-\frac{1}{2 \hbar} \Theta_{i j}(t) p_{j}, \quad p_{i}^{\mathrm{nc}}=p_{i}+\frac{1}{2 \hbar} \eta_{i j}(t) x_{j},
$$

with

$i, j=1,2 \ldots n$.
For a 2-dimensional noncommutative phase-space and according to Eq. (11), it becomes [38]

$$
\begin{aligned}
& x^{\mathrm{nc}}(t)=x-\frac{1}{2 \hbar} \Theta e^{\gamma t} p_{y}, \quad p_{x}^{\mathrm{nc}}(t)=p_{x}+\frac{1}{2 \hbar} \eta e^{-\gamma t} y \\
& y^{\mathrm{nc}}(t)=y+\frac{1}{2 \hbar} \Theta e^{\gamma t} p_{x}, \quad p_{y}^{\mathrm{nc}}(t)=p_{y}-\frac{1}{2 \hbar} \eta e^{-\gamma t} x .
\end{aligned}
$$

For $\gamma=0$, the time-dependency in the structure of the noncommutative parameter is canceled. When $\Theta=\eta=0$, the systems of noncommutative phasespace (62) and time-dependent noncommutative phasespace(64) will become commutative systems. We would like to note that if we have a problem of a charged particle in an electromagnetic field it would be quite well to approach it via the Seiberg-Witten maps. On the other hand, if we have a magnetic field interaction (or a problem of a quantum neutral particle moving under the action of a Newtonian gravitational potential for instance), we will follow a quite standard approach, that has been widely used in the literature on noncommutative quantum mechanics, which depends on obtaining a noncommutative version of a given field theory through the Moyal-Weyl product. 


\section{CONCLUSION}

In conclusion, the techniques described in this paper have proved to be invaluable methods for the study of noncommutative quantum mechanics and noncommutative field theory. As remarked in this paper, the noncommutativity is ordinarily formulated and coded mainly in terms of four methods, which are: (i) the ordinary product with Weyl operators, (ii) by replacing the ordinary product with the product of Moyal-Weyl in the functions and actions of the systems, (iii) using Bopp-shift linear transformations, (iv) through the maps of Seiberg-Witten. It is also worth clarifying that the noncommutativity could be time-dependent; we explained the reasons behind this, besides we extracted a time-dependent noncommutative algebra through noncommutative parameters considered exponential functions. Of course, we confirm that there are other options for noncommutative parameters forms.

Acknowledgments. The author would like to thank the anonymous reviewer for his careful reading of the manuscript and for his insightful comments and suggestions.
[1] H. S. Snyder, Phys. Rev. 71, 38 (1947); https://doi.or g/10.1103/PhysRev.71.38.

[2] H. S. Snyder, Phys. Rev. 72, 68 (1947); https://doi.or g/10.1103/PhysRev.72.68.

[3] A. Connes, Publ. Math. IHÉS 62, 41 (1985); https:// doi.org/10.1007/BF02698807.

[4] S. L. Woronowicz, Publ. Res. Inst. Math. Sci. 23, 117 (1987); https://doi.org/10.2977/prims/1195176848.

[5] S. L. Woronowicz, Commun. Math. Phys. 111, 613 (1987); https://doi.org/10.1007/BF01219077.

[6] N. Seiberg, E. Witten, J. High Energy Phys. 1999(09), 032 (1999); https: //doi .org/10.1088/1126-6708/1999 /09/032.

[7] T. Banks, W. Fischler, S. Shenker, L. Susskind, Phys. Rev. D 55, 5112 (1997); https://doi.org/10.1103/Ph ysRevD.55.5112.

[8] V. V. Nesvizhesky et al., Phys. Rev. D 67, 102002 (2003); https://doi.org/10.1103/PhysRevD.67.102002.

[9] V. V. Nesvizhesky et al., Nature 415, 297 (2002); https : //doi.org/10.1038/415297a.

[10] S. M. Carroll, J. A. Harvey, V. A. Kostelecký, C. D. Lane, T. Okamoto, Phys. Rev. Lett. 87, 141601 (2001); https : //doi.org/10.1103/PhysRevLett.87.141601.

[11] O. Bertolami, J. G. Rosa, C. M. L. de Aragão, P. Castorina, D. Zappalà, Phys. Rev. D 72, 025010 (2005): https: //doi.org/10.1103/PhysRevD.72.025010.

[12] I. Haouam, J. Phys. Stud. 24, 1801 (2020); https://do i.org/10.30970/jps. 24.1801.

[13] R. J. Szabo, Phys. Rep. 378, 207 (2003); https://doi. org/10.1016/S0370-1573(03)00059-0.

[14] I. Haouam, Open J. Microphys. 9, 15 (2019); https: //doi.org/10.4236/ojm. 2019.93003.

[15] J. Kamoshita, Eur. Phys. J. C 52, 451 (2007); https: //doi.org/10.1140/epjc/s10052-007-0371-y.

[16] S. Dey et al., Phys. Rev. D 90, 084005 (2014);https: //doi.org/10.1103/PhysRevD.90.084005.

[17] F. Delduc et al., J. Phys.: Conf. Ser. 103, 012020 (2008); https://doi.org/10.1088/1742-6596/103/1/012020.

[18] H. Weyl, The Theory of Groups and Quantum Mechanics (Dover, New York, 1931).

[19] M. A. Rieffel, Mem. Am. Math. Soc. 506, 106 (1993).

[20] S. M. R. Al-Btoush, Int. J. Novel Res. Phys. Chem.
Math. 3(3), 18 (2016).

[21] M. J. Schraml et al., Eur. Phys. J. C 16, 161 (2000); https://doi.org/10.1007/s100520050012.

[22] F. Schwabl, Quantum Mechanics (Springer, Berlin, 1995).

[23] A. Van-Brunt, M. Visser, Mathematics 6, 135 (2018); https://doi.org/10.3390/math6080135.

[24] M. Najafizadeh, M. Saadat, Chin. J. Phys. 51, 94 (2013); https://doi .org/10.6122/CJP.51.94.

[25] I. Haouam, Symmetry 11, 223 (2019); https://doi.or $\mathrm{g} / 10.3390 /$ sym 11020223 .

[26] I. Haouam, L. Chetouani, J. Mod. Phys. 9, 2021 (2018); https://doi.org/10.4236/jmp.2018.911127.

[27] Li Kang, D. Sayipjamal, Chin. Phys. C 34, 944 (2010); https://doi .org/10.1088/1674-1137/34/7/003.

[28] Sh. Minwalla et al., J. High Energy Phys. 2000(02), 020 (2000); https : //doi .org/10.1088/1126-6708/2000 /02/020.

[29] M. M. Sheikh-Jabbari, Phys. Rev. Lett. 84, 5265 (2000); https://doi.org/10.1103/PhysRevLett.84.5265.

[30] B. Jurčo, L. Möller, S. Schraml, P. Schupp, J. Wess, Eur. Phys. J. C 21, 383 (2001); https://doi.org/10.1007/ s100520100731.

[31] J. B. Schraml et al., Eur. Phys. J. C 17, 521 (2000);ht tps://doi.org/10.1007/s100520000487.

[32] S. Fidanza, Thèse de doctorat (École Polytechnique X, 2003); https://tel .archives-ouvertes.fr/pastel-00 000709.

[33] X. Calmet et al., Eur. Phys. J. C 23, 363 (2002); https: //doi.org/10.1007/s100520100873.

[34] J. Trampetić, M. Wohlgenannt, Phys. Rev. D 76, 127703 (2007); https://doi.org/10.1103/PhysRevD.7 6.127703 .

[35] K. Li, J. Wang, C. Chen, Mod. Phys. Lett. A 20, 2165 (2005);https://doi.org/10.1142/S0217732305017421.

[36] C. Bastos, O. Bertolami, Phys. Lett. A 372, 5556 (2008); https://doi.org/10.1063/1.2944996.

[37] S. Gangopadhyay, A. Saha, A. Halder, Phys. Lett. A 379, 2956 (2015); https://doi.org/10.1016/j.physle ta. 2015.08.039.

[38] I. Haouam, preprint arXiv:1910.05833v1 (2019). 


\title{
ПРО НЕКОМУТАТИВНУ ГЕОМЕТРІЮ У КВАНТОВІЙ МЕХАНІЦІ
}

\author{
Ільяс Гавам \\ Університет імені братів Ментурі, Константина, Алюир
}

У статті наведено огляд формалізму, який відіграє центральну роль у більшості досліджень, що стосуються некомутативної геометрії. Описано наявні методи, які успішно дозволяють використовувати та застосовувати некомутативність фазового простору як у квантовій механіці, так й у квантовій теорії поля. Зокрема, коротко пояснено квантування Вейля, добуток Мояла-Вейля, перетворення зсуву Боппа та відображення Зайберга-Віттена.

Ключові слова: некомутативна геометрія, некомутативна квантова механіка, квантування Вейля, добуток Мояла-Вейля, перетворення зсуву Боппа, відображення Зайберга-Віттена, відображення ВейляВігнера, співвідношення невизначеностей. 